\title{
Aerodynamic Shape Optimization of Aircraft Wings Using Panel Methods
}

\author{
Conlan-Smith, Cian; Ramos-García, Néstor; Sigmund, Ole; Andreasen, Casper Schousboe
}

Published in:

AIAA Journal

Link to article, DOI:

10.2514/1.J058979

Publication date:

2020

Document Version

Peer reviewed version

Link back to DTU Orbit

Citation (APA):

Conlan-Smith, C., Ramos-García, N., Sigmund, O., \& Andreasen, C. S. (2020). Aerodynamic Shape

Optimization of Aircraft Wings Using Panel Methods. AIAA Journal, 58(9). https://doi.org/10.2514/1.J058979

\section{General rights}

Copyright and moral rights for the publications made accessible in the public portal are retained by the authors and/or other copyright owners and it is a condition of accessing publications that users recognise and abide by the legal requirements associated with these rights.

- Users may download and print one copy of any publication from the public portal for the purpose of private study or research.

- You may not further distribute the material or use it for any profit-making activity or commercial gain

- You may freely distribute the URL identifying the publication in the public portal

If you believe that this document breaches copyright please contact us providing details, and we will remove access to the work immediately and investigate your claim. 


\title{
On Aerodynamic Shape Optimization of Aircraft Wings using Panel Methods
}

\author{
Cian Conlan-Smith *, Néstor Ramos-García ${ }^{\dagger}$, Ole Sigmund ${ }^{\star}$ and Casper Schousboe Andreasen ${ }^{\S}$ \\ Technical University of Denmark, DK-2800 Lyngby, Denmark.
}

Panel methods are frequently applied to aerodynamic shape optimization problems due to their fast turnaround time and ability to model arbitrary geometries. Despite being advantageous for design optimization, we have found that panel methods can predict non-physical results for unconventional geometries. This work presents robust methods to solve optimization problems using panel methods that are not susceptible to numerical errors. Important factors are highlighted with regard to choice in boundary conditions, induced drag calculation, wake modeling, and regularization. Two parameterization methods are introduced where wing geometry is defined locally by airfoils at discrete span-wise positions and regularized by filtering along the span. Such methods of defining the geometry locally, enlarge the design space and allow the optimizer to converge to reliable designs. Results also suggest that: enforcing a Dirichlet boundary condition rather than a Neumann formulation provides significant cost savings in gradient calculations; far-field force calculations should be adopted for optimization problems as numerical errors in surface pressure integration have a strong influence on the gradients; and the additional design freedom of a B-spline parameterization can be disadvantageous as the low-fidelity of the inviscid model cannot correctly capture aerodynamic properties of irregular airfoil geometries.

\section{Nomenclature}

$$
\begin{aligned}
& \boldsymbol{A}=\text { doublet aerodynamic influence coefficient } \\
& \boldsymbol{B}=\text { source aerodynamic influence coefficient } \\
& b \quad=\text { wingspan } \\
& c \quad=\text { chordlength } \\
& D \quad=\text { total drag } \\
& \boldsymbol{F} \quad=\text { total force }
\end{aligned}
$$

\footnotetext{
*PhD student, Department of Mechanical Engineering, Section of Solid Mechanics, cicosm@ mek.dtu.dk. Member AIAA.

${ }^{\dagger}$ Senior Researcher, Department of Wind Energy, Section of Fluid Mechanics.

\$Professor, Department of Mechanical Engineering, Section of Solid Mechanics.

${ }^{\S}$ Associate Professor, Department of Mechanical Engineering, Section of Solid Mechanics.
} 


$$
\begin{aligned}
& f \quad=\text { generic function } \\
& L=\text { total Lift } \\
& M_{\infty}=\text { free-stream Mach number } \\
& M_{r} \quad=\text { root bending moment } \\
& M_{p}=\text { pitching moment } \\
& m=\text { maximum relative camber } \\
& n=\text { normal vector } \\
& \boldsymbol{P}=\text { coordinates of control points } \\
& p \quad=\text { relative position of maximum camber } \\
& R=\text { filter radius } \\
& S \quad=\text { wing planform area } \\
& t=\text { maximum relative thickness } \\
& U=\text { total velocity } \\
& X=\text { coordinates } \\
& \alpha=\text { twist } \\
& \beta=\sqrt{1-M_{\infty}^{2}} \text {, transformation factor } \\
& \delta \quad=\text { design variables } \\
& \eta \quad=\frac{2 y}{b} \text {, normalized span location } \\
& \kappa=\text { curvature } \\
& \lambda=\text { Lagrange multiplier } \\
& \mu \quad=\text { doublet strength } \\
& \phi \quad=\text { potential function } \\
& \rho \quad=\text { density } \\
& \sigma \quad=\quad \text { source strength } \\
& \mathbb{R}=\frac{b^{2}}{S} \text {, aspect ratio } \\
& C_{P}=\text { pressure coefficient } \\
& \mathcal{F}=\text { generic function in Lagrangian form } \\
& \boldsymbol{W}=\text { filter matrix }
\end{aligned}
$$

\section{Subscripts}

$D=$ associated with Dirichlet boundary condition

$N=$ associated with Neumann boundary condition

ref $=$ associated with reference case 


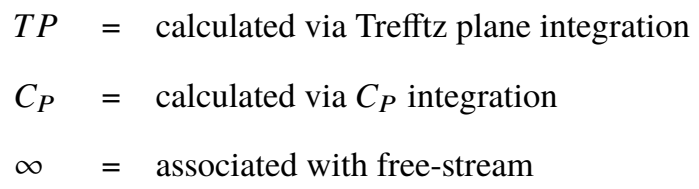

\section{Introduction}

Panel methods were developed in the 1960s and 70s within the aviation industry for evaluating the performance of preliminary aircraft designs [1-3]. Based on potential flow theory these methods provide the velocity field and pressure distribution around arbitrary geometries. As boundary element methods, panel methods only require a surface mesh of the geometry, leading to a fast computational time when compared to computational fluid dynamics (CFD) based on finite volume and finite element methods, which require a volume mesh of the entire fluid domain. This makes panel methods attractive for aerodynamic optimization where a solution to the physics problem is required for each design iteration. Additionally, as only a surface mesh is used, it is easier to avoid mesh distortion or remeshing, which is common in shape optimization with CFD.

In the 1970 s researchers began to investigate the design of airfoils through numerical optimization. In a series of works by Hicks et al. [4 6] airfoils were parametrized using high order polynomials or Joukowski transformations. Venkataraman [7] introduced the idea of using splines to parametrize the airfoil where two Bézier curves were used to define upper and lower surfaces, and have since been used to define thickness and camber distributions [8, 9]. Basis splines (B-splines) were later adopted for similar parameterization methods [10 12] as they are less susceptible to bumps or fluctuations because the order of the curve is not defined by the number of points. Consequently, B-splines have shown to achieve better off-design performance when compared to Bézier curves [13-15]. B-spline representations have since become the most popular parametrization method for airfoil optimization [16-[18]. However in recent times, parameterizing using free-form deformation (FFD) techniques has also gained much popularity [19, 20].

The first automated design processes for 3D wings using panel methods involved solving inverse problems [21, 22] where the wing geometry is achieved such that it produces a user specified pressure distribution. However, it can be difficult to specify a target pressure distribution that creates the desired or optimal performance. By the end of the 1990s CFD methods had already started to be applied to more general 3D aerodynamic [23, 24] and multidisciplinary optimization (MDO) problems [25]. The growing interest in optimization studies saw the need for fast calculations of aerodynamic loads, where panel methods are effective. Choi et al. [26, 27] created a framework for multi-fidelity optimization with the ability to solve the Euler equations and linearized potential flow problems via a panel method (PanAir [28]). Similar gradient-free studies also implementing PanAir were conducted by Alonso et al. [29], and Rahnarayan et al. [30] who implemented PanAir as the high-fidelity method and an area rule method as a low-fidelity method. Kennedy and Martins demonstrated a parallel framework for aero-elastic MDO using a panel method (TriPan) 
and finite element (FE) solver [31, 32]. TriPan has been applied to a number of subsequent aero-structural optimization problems such as the design of composite wings [33], wing-box topology optimization [34, 35], matrix-free optimization [36], and unsteady problems [37, 38]. Goetzendorf-Grabowski and Mieloszyk [39, 40] present another coupled panel-FE framework for MDO based on the open-source PANUKL code.

The aim of this work is to firstly highlight the main considerations and challenges in using panels methods in optimization problems, and secondly to introduce novel parametrization methods for aerodynamic optimization problems that allow large changes in geometry. Despite there being interest in panel methods for optimization problems the literature lacks detailed comparisons and discussions of their application. Approached from an optimization perspective, external Neumann and internal Dirichlet boundary conditions are compared in their performance with respect to computational time, ability to predict solutions, and applicability to optimization problems. Challenges have been found in applying these methods to optimization problems with regard to choice of wake model and force calculation, especially when the parameterization methods allow large changes in the geometry. Traditionally, wing optimization problems have been parameterized using global variables such as a taper ratio and predefined airfoils. Using such global variables to define the geometry restricts the design space which can lead to sub-optimal designs. We introduce free-form parameterization approaches where airfoil sections are defined locally and design variables are filtered along the span for regularization and avoidance of numerical artifacts.

The current work uses a subsonic constant source-doublet panel method based on MIRAS [41]. An introduction to the panel method is detailed in Section $\Pi$ covering the problem formulation, wake modeling and aerodynamic force calculations. Section III] describes the mesh discretization and two parameterization methods which are later compared. The optimization approach is detailed in Section IV] including the formulation of the optimization problem, regularization, and sensitivity analysis. The results section (Section $\mathrm{V}$ ) discusses the choice of boundary condition, wake model, and force calculation method with respect to their performance and applicability to optimization problems. Optimization results are also presented for two parameterization methods and compared for their performance and ability to predict realistic designs. Finally, the findings are concluded in Section VI Additionally, the appendix includes verification studies with comparisons to lifting-line theory, VSAERO panel code, and SU2 finite volume method.

\section{Panel Method}

In this work we implement a combined source and doublet panel method with a Prantl-Glauert compressibility correction [42, 43]. We shortly repeat the basic theory here to provide a basis for later discussions and comparisons. A typical panel method discretization is shown in Fig. 1 with collation points defined at the center of each panel. Both Neumann and Dirichlet boundary conditions are considered and are based on a zero internal perturbation formulation 
[44, 45]. The governing equations are defined as

$$
\begin{gathered}
\mathbf{A}_{N} \boldsymbol{\mu}+\left(\mathbf{B}_{N}+\mathbf{I}\right) \sigma=\mathbf{0} \\
\mathbf{A}_{D} \boldsymbol{\mu}+\mathbf{B}_{D} \boldsymbol{\sigma}=\mathbf{0}
\end{gathered}
$$

where $\mathbf{A}$ and $\mathbf{B}$ are aerodynamic influence coefficients (AIC) for doublet and source distributions respectively. Subscripts $N$ and $D$ represent AIC for Neumann and Dirichlet boundary conditions, i.e. the influence on the velocity or potential function at a collocation point. Influence coefficients are derived using the methods outlined in [46], Section 10.4. It is important to note that each panel will influence every other point in the domain, meaning that $\mathbf{A}$ and $\mathbf{B}$ are dense matrices. For a zero internal perturbation formulation the source strengths, $\sigma$, are calculated using via

$$
\sigma_{i}=\boldsymbol{U}_{\infty} \cdot \boldsymbol{n}_{i}
$$

and equation (1) is used to solve for doublet strengths, $\boldsymbol{\mu}$.

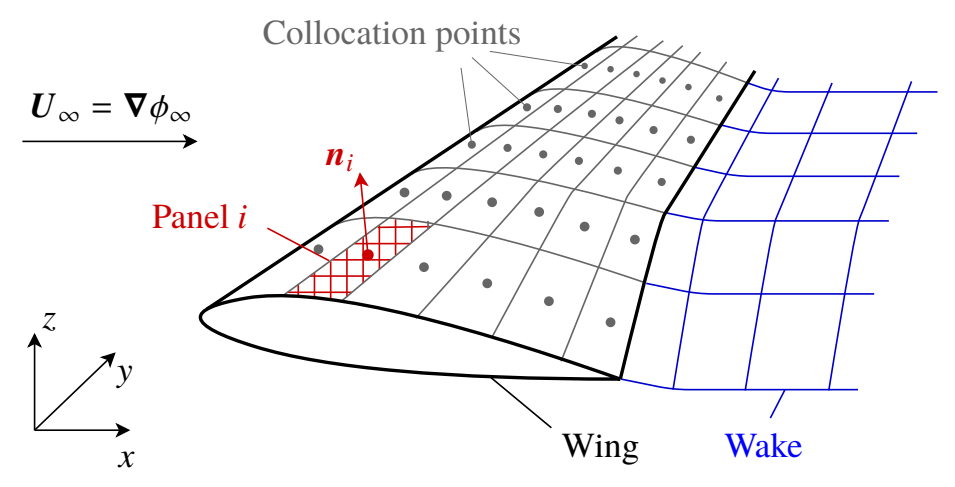

Fig. 1 Schematic of a wing and its wake discretized into quadrilateral panels.

As shown in Fig. 1 1 the wing's wake is also meshed which is required to satisfy the Kutta condition and calculate the induced drag. Two wake models are compared in this work, the first is a fixed wake consisting of a vortex sheet projected in the free-stream direction. This model is computationally efficient because of a low number of panels and governing equations are only solved once. However, the method may become inaccurate for wing geometries that create a large variance in the structure of the wake. An alternative is a force-free wake model [41, 47, 48] which is an iterative procedure that is able to capture complicated wake geometries, but requires (1) to be solved on each iteration leading to a large computational expense.

Once the singularity distribution is known, the velocity at each panel's collocation point can be calculated as the 
gradient of the potential function in local coordinates $(l, m, n)$.

$$
\boldsymbol{U}_{i}=\left(U_{l}, U_{m}, U_{n}\right)_{i}^{T}=\left(\frac{\partial \phi}{\partial l}, \frac{\partial \phi}{\partial m}, \frac{\partial \phi}{\partial n}\right)_{i}^{T}
$$

The pressure coefficient, $C_{P}$, is calculated via the Bernoulli equation (4), and can be integrated over the body surface to find the total force, $\boldsymbol{F}$, pitching moment, $M_{p}$, and root bending moment, $M_{r}$.

$$
\begin{gathered}
C_{P, i}=1-\frac{\left\|\boldsymbol{U}_{i}\right\|^{2}}{\left\|\boldsymbol{U}_{\infty}\right\|^{2}} \\
\boldsymbol{F}=\frac{1}{2} \rho_{\infty} U_{\infty}^{2} \int_{S} C_{P} \boldsymbol{n} d S \simeq \frac{1}{2} \rho_{\infty} U_{\infty}^{2} \sum_{i=1}^{N_{S}} C_{P, i} S_{i} \boldsymbol{n}_{i} \\
M_{p}=\frac{1}{2} \rho_{\infty} U_{\infty}^{2} \int_{S} C_{P}\left\{\begin{array}{l}
z \\
x
\end{array}\right\}^{\mathrm{T}}\left\{\begin{array}{l}
n_{x} \\
n_{z}
\end{array}\right\} d S \simeq \frac{1}{2} \rho_{\infty} U_{\infty}^{2} \sum_{i=1}^{N_{S}} C_{P, i} S_{i}\left\{\begin{array}{l}
z \\
x
\end{array}\right\}_{i}^{\mathrm{T}}\left\{\begin{array}{l}
n_{x} \\
n_{z}
\end{array}\right\}_{i} \\
M_{r}=\frac{1}{2} \rho_{\infty} U_{\infty}^{2}\left\|\int_{S} C_{P} y\left\{\begin{array}{l}
n_{x} \\
n_{z}
\end{array}\right\} d S\right\| \simeq \frac{1}{2} \rho_{\infty} U_{\infty}^{2}\left\|\sum_{i=1}^{N_{S}} C_{P, i} S_{i} y_{i}\left\{\begin{array}{l}
n_{x} \\
n_{z}
\end{array}\right\}_{i}\right\|
\end{gathered}
$$

where $x_{i}, y_{i}$, and $z_{i}$ are coordinates of panel $i$ 's collocation point (with the origin at the root). Lift and induced drag are defined by projecting $\boldsymbol{F}$ perpendicular and parallel to the free-stream direction, which in our case is always in the $x$-direction

$$
\begin{aligned}
L_{C_{P}} & =\boldsymbol{F} \cdot \mathbf{z} \\
D_{C_{P}} & =\boldsymbol{F} \cdot \mathbf{x}
\end{aligned}
$$

where $D_{C_{P}}$ and $L_{C_{P}}$ are the forces calculated via $C_{P}$-integration. Alternatively, the forces can be calculated through far-field calculations using a Trefftz plane integration method, $L_{T P}$ and $D_{T P}$ [43, 49]. This reduces lift and drag calculations to a 2D integral over the intersection of the wake and the Trefftz plane, $s_{w}$ (refer to Fig. 2).

$$
\begin{gathered}
L_{T P}=\rho_{\infty} V_{\infty} \int_{s_{w}} \Delta \phi d y \simeq \rho_{\infty} V_{\infty} \sum_{i=1}^{N_{w}} \mu_{i} s_{i} \cos \left(\theta_{i}\right) \\
D_{T P}=-\frac{1}{2} \rho_{\infty} \int_{s_{w}} \Delta \phi \frac{\partial \phi}{\partial n} d s_{w} \simeq-\frac{1}{2} \rho_{\infty} \sum_{i=1}^{N_{w}} \mu_{i} s_{i} \boldsymbol{u}_{i} . \boldsymbol{n}_{i}
\end{gathered}
$$

where $\Delta \phi$ is the potential jump over $s_{w}, \theta_{i}$ is the inclination of wake panel $i$ in the Trefftz plane, $\boldsymbol{n}$ is normal to $s_{w}$ in the Trefftz plane.

Induced drag is the only type of drag considered in this work. Aerodynamic loads can be normalized to find 


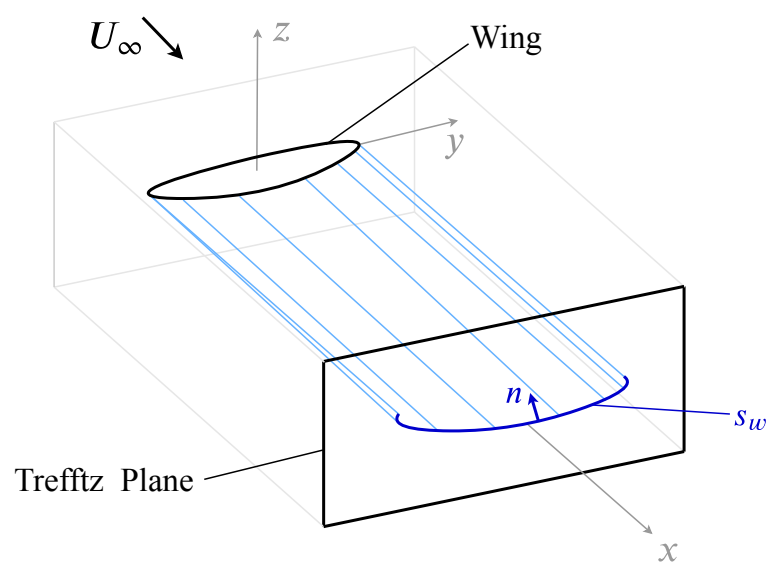

Fig. 2 Schematic of the Trefftz plane integration method.

coefficients of lift and drag.

$$
\begin{aligned}
C_{L} & =\frac{L}{1 / 2 \rho_{\infty} U_{\infty}^{2} S} \\
C_{D} & =\frac{D}{1 / 2 \rho_{\infty} U_{\infty}^{2} S}
\end{aligned}
$$

If the Prandtl-Glauert correction is used, the quantities in equations (4)-(13) are first calculated using coordinates from Göthert's transformation [43, 50], yielding quantities denoted with the subscript ${ }_{0}$ below, and are then corrected using the Prandtl-Glauert factor, $\beta=\sqrt{1-M_{\infty}^{2}}$.

$$
C_{P}=\frac{1}{\beta^{2}} C_{P, 0} \quad, \quad L=\frac{1}{\beta^{3}} L_{0} \quad, \quad D=\frac{1}{\beta^{4}} D_{0} \quad, \quad C_{L}=\frac{1}{\beta^{2}} C_{L_{0}} \quad, \quad C_{D}=\frac{1}{\beta^{3}} C_{D_{0}}
$$

\section{Discretization and Parametrization}

The wing geometry is discretized into quadrilateral panels where nodal points are typically defined to be equallyspaced in the span-wise direction, and in a cosine distribution in the chord-wise direction creating a bias towards leading and trailing edges. This bias allows more detail in the $C_{P}$ distributions making the $C_{p}$-integration less prone to numerical error. The location of nodes in the $x$ - and $z$-directions are given by airfoil geometries defined at $N_{s}$ span-wise locations each with $N_{a f}$ nodes. Airfoil sections are defined in one of two ways - using the definition of NACA airfoils, and B-spline representations. Half the span is modeled, without a fuselage, and symmetry is enforced by modifying influence coefficients to include an influence from the wing's mirror image about the root. Unless otherwise stated a mesh of 6000 (40 span-wise, 150 chord-wise) panels was used for a single wing.

When using quadrilateral panels it is not guaranteed that the four nodes will lie in the same plane, as it takes only three points to define a plane. The panel is instead defined using an average plane as described in [51]. The end 
sections of the wing are also meshed creating an enclosed geometry. When implementing a panel method the problem is ill-defined for an enclosed mesh that separates internal and external domains with doublet panels [52]. There are multiple ways around this such as defining an additional source panel with some known potential in the internal domain, or only specifying source panels (i.e. non-lifting panels) in certain locations. We have adopted the latter where the wing's end-caps consist of source only panels.

\section{A. NACA parameterization}

This parameterization is based on the polynomial equations for defining NACA 4- and 5-digit airfoils [53], where 4 parameters are used (refer to Fig. 3): chord length $c$, maximum thickness $t$, maximum camber $m$, and position of maximum camber $p$. However, note that NACA 5-digit airfoils do not require both $m$ and $p$ to be defined, and the ideal lift coefficient (first coefficient) is specified. The NACA 4- and 5-digit airfoil family consists of a set of airfoils formed from discrete values of these parameters. Our parameterization differs in that the design space is smoothed such that the optimizer can select any value for these parameters from a continuous design space. Additionally, thickness is traditionally defined perpendicular to the camber line, however, using this definition, the derivatives are discontinuous at the point of maximum camber which may cause issues in the optimization. To avoid this we take the thickness to be perpendicular to the chord line (see Fig. 3). The twist, $\alpha$, is included as an additional variable, giving the NACA parameterization a possible 5 design variables per airfoil section. The wing coordinates, $\boldsymbol{X}$, are achieved by rotating airfoil coordinates, $(\bar{x}, \bar{z})$, through $\alpha$ and assembling airfoil sections with their quarter chord point on the $y$-axis.

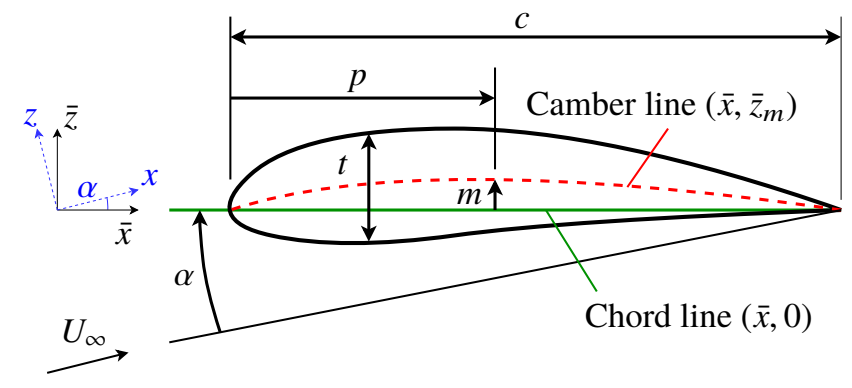

Fig. 3 Design variables used in the NACA parameterization: $c=$ chord length, $t=$ maximum thickness, $m=$ maximum camber, $p=$ position of maximum camber, and $\alpha=$ twist.

\section{B. B-spline parameterization}

Here the wing coordinates, $\boldsymbol{X}$, are given by a series of airfoils represented as B-spline curves with control points $\mathbf{P}$ (definitions of B-splines are presented in [54]). In our B-spline parameterization, represented in Fig. 4, the relative spacings between control points in the $x$-direction are kept constant, where all control points scale in the $x$-direction about the trailing edge, which is controlled by a single design variable per section. Control points are then free to move vertically independent of one another as controlled by one design variable per control point. The spline is clamped so 
that it starts and ends at the first and last control point which are coincident. The wing is assembled from each airfoil section with a common axis at the quarter chord point. Bounds are imposed on each degree of freedom in the $z$-direction such that the spline does not self-intersect. If a control point approaches one of these bounds and the other control point at the same $x$-coordinate is not nearby (i.e. the airfoil is not collapsing), then the bound is updated to enlarge the range. When the bounds are defined or updated they are specified at a small distance from the camber line of the design on the current iteration.

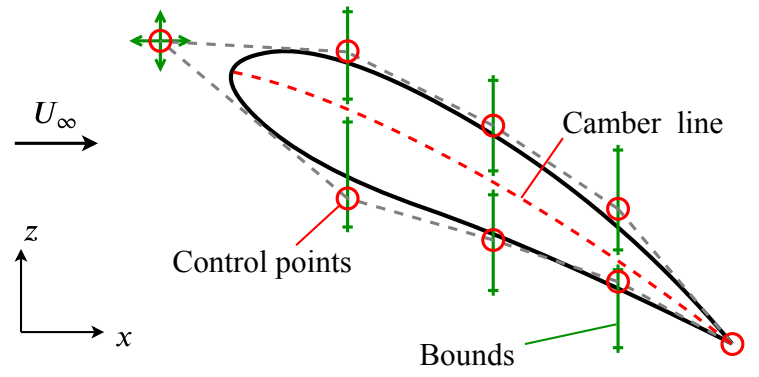

Fig. 4 B-spline airfoil parametrization with control points (red circles), their degrees of freedom/bounds (green), and camber line (dashed red) shown.

\section{Optimization approach}

\section{A. Physical considerations when optimizing lifting surfaces}

Typically optimization problems will be posed using absolute quantities, such as $L$ and $D$, rather than non-dimensional quantities, such as $C_{L}$ and $C_{D}$, as they are generally more stable for the optimization problem. For example, consider a minimum constraint on $C_{L}$. The value of $C_{L}$ can be continually increased by shrinking the planform area (refer to (12) where a minimum bound on $S$ is required to prevent the problem being ill-posed. Oppositely, for a minimum constraint on $L$ there should exist an optimum design where the constraint is active and the area of the wing has not reduced to a non-physical size. The most important constraint is the minimum bound on $L$, as induced drag is dependent on lift, there will be no induced drag for $L=0$ which is a trivial optimal solution.

\section{B. Span-wise regularization}

Regularization is necessary to prevent clustering of nodes and drastic variations in geometry which may be non-physical, non-manufacturable, or cause numerical issues [55, 56]. Design variables are applied at a discrete set of airfoil sections with a single panel between sections. A large variation in geometry between sections cannot be accurately represented by a single panel and leads to non-physical results which the optimizer is able to exploit. Filtering is used to control the variation between sections where the filter size can act as a feature control parameter.

Design variables are filtered based on the span-wise positions of neighboring sections. A filter radius, $R$, is defined 
such that a neighboring section, $j$, will have a weight proportional to its span-wise distance if the distance between sections is less than $R$, and carry no weight if the distance is greater than $R$. This is similar to a density filter commonly adopted in topology optimization [57]. Weights are stored within the filter matrix, $\boldsymbol{W}$, the independent design variables are contained within $\boldsymbol{\delta}$, and the filtered or physical design variables, $\tilde{\boldsymbol{\delta}}$, are defined as

$$
\tilde{\delta}=\boldsymbol{W} \delta
$$

where

$$
\mathcal{W}_{i j}=\frac{1}{\sum_{k=1}^{N_{s}} w_{i k}} w_{i j} \text { where } w_{i j}=\max \left[0, R-d_{i j}\right]
$$

For the NACA parameterization design variables are defined as $\delta=\{\boldsymbol{c}, \boldsymbol{t}, \boldsymbol{m}, \boldsymbol{p}, \boldsymbol{\alpha}\}$ where each airfoil parameter is filtered along the span. For the B-spline parameterization the design variables are the coordinates of the B-spline control points, $\boldsymbol{\delta}=\mathbf{P}$. The coordinates of control points $i$ (where $i=1, \ldots, n+1$ for each airfoil section, $j$ ) are filtered along the span using the same method as the NACA parameterizations.

\section{Airfoil regularization}

As the flow is inviscid the method is unable to predict flow separation. The optimizer is able to exploit this deficiency through creating sharp edges or bumped surfaces which reduce the objective function but lead to unrealistic pressure distributions. To prevent this, a constraint on the maximum curvature is used. For the NACA parameterization the maximum curvature is always at the leading edge of the airfoil. Curvature is related to radius by $\kappa=1 / r$, where the leading edge radius for any NACA airfoil is approximated as

$$
r_{\mathrm{LE}}=1.1019 t^{2} c
$$

We wish to constrain the normalized maximum curvature, $\hat{\kappa}$, that is the maximum curvature of the airfoil whose coordinates are scaled between 0 and 1 in the $x$-direction, yielding

$$
\hat{\kappa}_{j}=\frac{1}{1.1019 t_{j}^{2}}
$$

For the B-spline parameterization curvature is first calculated at each node, $i$, on the spline.

$$
\kappa_{i}=\frac{x_{i}^{\prime} z_{i}^{\prime \prime}-z_{i}^{\prime} x_{i}^{\prime \prime}}{\left[\left(x_{i}^{\prime}\right)^{2}+\left(z_{i}^{\prime}\right)^{2}\right]^{\frac{3}{2}}}
$$

The maximum curvature is then approximated by aggregating $\kappa$ using a p-norm function and similar to the NACA 
parameterizations is normalized by $c_{j}$ to give $\hat{\kappa}_{j}$ for airfoil section $j$

$$
\hat{\kappa}_{j}=c_{j}\left[\sum_{i=1}^{N_{a f}} \kappa_{i}^{\zeta}\right]^{\frac{1}{\zeta}}
$$

where $\zeta$ is taken to be equal to 10 throughout.

\section{Sensitivity Analysis}

The method of moving asymptotes (MMA) [58] is used as the optimizer which requires sensitivities to determine how design variables should change between iterations of the optimization procedure. Here, an adjoint method is used for calculating sensitivities. The objective or constraint function of interest, $f$, can be expressed in augmented Lagrangian form as

$$
\mathcal{F}=f+\lambda^{T} \mathbf{R}
$$

where $\lambda$ is the Lagrange multiplier, and $\mathbf{R}$ is the residual to the state equation given in $(1 \mathrm{a})$ and $(1 \mathrm{~b})$ for Neumann and Dirichlet BCs respectively. Note that $\lambda$ is arbitrary as $\mathbf{R}=\mathbf{0}$, thus $\mathcal{F}=f$ for any $\lambda$. To conduct shape optimization we require the sensitivities of $\mathcal{F}$ with respect to the nodal coordinates, $\boldsymbol{X}$. Differentiating 21 with respect to nodal coordinates and expanding using the chain rule yields

$$
\frac{d \mathcal{F}}{d \boldsymbol{X}}=\frac{\partial f}{\partial \boldsymbol{X}}+\frac{\partial f}{\partial \boldsymbol{\mu}} \frac{d \boldsymbol{\mu}}{d \boldsymbol{X}}+\lambda^{T}\left[\frac{\partial \mathbf{R}}{\partial \boldsymbol{X}}+\frac{\partial \mathbf{R}}{\partial \boldsymbol{\mu}} \frac{d \boldsymbol{\mu}}{d \boldsymbol{X}}\right]
$$

Note the difference between partial, $\partial / \partial \boldsymbol{X}$ and total, $d / d \boldsymbol{X}$ derivative operators, where partial derivatives capture only the explicit dependence without resolving the state equation, whereas total derivatives capture implicit dependencies also. Implicit dependencies are difficult to calculate and as such we seek a value of $\lambda$ which causes all total derivatives to vanish. Equation (22) can be re-expressed as

$$
\frac{d \mathcal{F}}{d \boldsymbol{X}}=\frac{\partial f}{\partial \boldsymbol{X}}+\lambda^{T} \frac{\partial \mathbf{R}}{\partial \boldsymbol{X}}+\left[\frac{\partial f}{\partial \boldsymbol{\mu}}+\lambda^{T} \frac{\partial \mathbf{R}}{\partial \boldsymbol{\mu}}\right] \frac{d \boldsymbol{\mu}}{d \boldsymbol{X}}
$$

By setting terms in the square brackets in (23) equal to zero and solving for $\lambda$ the implicit derivatives are eliminated. This corresponds to solving the adjoint problem.

$$
\lambda^{T}=-\frac{\partial f}{\partial \mu}\left[\frac{\partial \mathbf{R}}{\partial \boldsymbol{\mu}}\right]^{-1}=-\frac{\partial f}{\partial \mu} \mathbf{A}^{-1}
$$

and thus it follows that

$$
\frac{d \mathcal{F}}{d \boldsymbol{X}}=\frac{\partial f}{\partial \boldsymbol{X}}+\lambda^{T} \frac{\partial \mathbf{R}}{\partial \boldsymbol{X}}
$$


Differentiating the state equation in $1 \mathrm{a}$ ) or $1 \mathrm{~b}$ ) with respect to the nodal coordinates yields the residual derivatives

$$
\begin{aligned}
& \frac{\partial \mathbf{R}}{\partial \boldsymbol{X}}=\frac{\partial \mathbf{A}_{N}}{\partial \boldsymbol{X}} \boldsymbol{\mu}+\frac{\partial \mathbf{B}_{N}}{\partial \boldsymbol{X}} \boldsymbol{\sigma}+\mathbf{B}_{N} \frac{\partial \boldsymbol{\sigma}}{\partial \boldsymbol{X}}+\frac{\partial \boldsymbol{\sigma}}{\partial \boldsymbol{X}} \\
& \frac{\partial \mathbf{R}}{\partial \boldsymbol{X}}=\frac{\partial \mathbf{A}_{D}}{\partial \boldsymbol{X}} \boldsymbol{\mu}+\frac{\partial \mathbf{B}_{D}}{\partial \boldsymbol{X}} \boldsymbol{\sigma}+\mathbf{B}_{D} \frac{\partial \boldsymbol{\sigma}}{\partial \boldsymbol{X}}
\end{aligned}
$$

where suffixes (a) and (b) are for Neumann and Dirichlet BCs respectively. Up to now sensitivities are calculated with respect to Göthert's transformed coordinates, $\boldsymbol{X}_{0}$. Using the chain rule sensitivities are transformed back to the real coordinates, then subsequently to physical design variables, $\tilde{\boldsymbol{\delta}}$, and finally independent design variables, $\boldsymbol{\delta}$, as defined by the parametrization method in Section III]

$$
\frac{d \mathcal{F}}{d \boldsymbol{\delta}}=\frac{d \mathcal{F}}{d \boldsymbol{X}_{0}} \frac{d \boldsymbol{X}_{0}}{d \boldsymbol{X}} \frac{d \boldsymbol{X}}{d \tilde{\boldsymbol{\delta}}} \frac{d \tilde{\boldsymbol{\delta}}}{d \boldsymbol{\delta}}=\frac{d \mathcal{F}}{d \boldsymbol{X}_{0}}\left[\begin{array}{ccc}
\mathbf{I} & 0 & \mathbf{0} \\
0 & \frac{1}{\beta} \mathbf{I} & \mathbf{0} \\
\mathbf{0} & \mathbf{0} & \frac{1}{\beta} \mathbf{I}
\end{array}\right] \frac{d \boldsymbol{X}}{d \tilde{\boldsymbol{\delta}}} \boldsymbol{W}
$$

where $\frac{d \mathcal{F}}{d \boldsymbol{X}_{0}}$ is calculated using [25], $\frac{d \boldsymbol{X}}{d \tilde{\boldsymbol{\delta}}}$ is the derivative of parameterization method defined in Section III] and $\boldsymbol{W}$ is the filter matrix defined in 16 .

\section{Results}

For the following optimization studies we define a reference wing with a rectangular planform, aspect ratio, $\mathbb{R}=6$ ( $b=6 \mathrm{~m}$ and $c=1 \mathrm{~m}$ ) and constant NACA0012 airfoils throughout the span. The performance characteristics of this wing are shown in Fig. 5. Optimized designs are compared to this wing's performance at Mach 0.4 with an angle of attack of $6^{\circ}$, induced drag, $D_{\text {ref }}=915.6 \mathrm{~N}$ and total lift, $L_{\text {ref }}=34525.4 \mathrm{~N}$. The properties of this wing also give the bounds for constraint functions in all optimization problems.

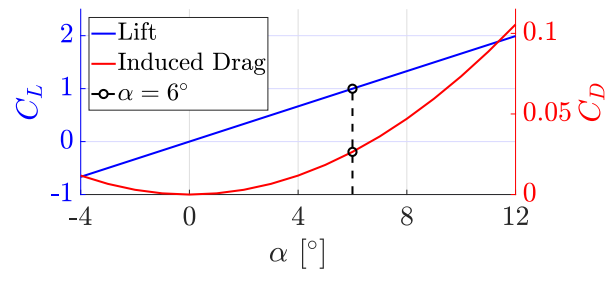

(a)

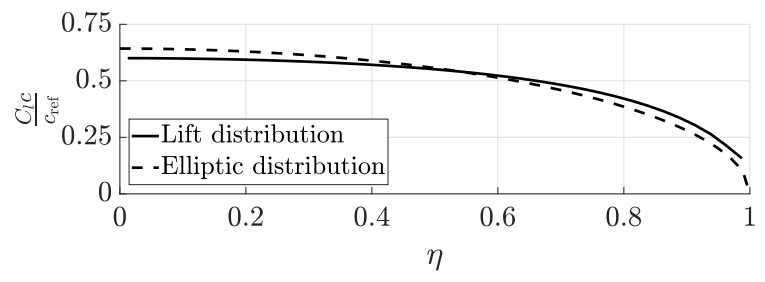

(b)

Fig. 5 Performance characteristics of the reference wing: (a) lift and induced drag coefficients for varying angles of attack, $\alpha$, and (b) normalized sectional lift area distribution along the half span for $\alpha=6^{\circ}$.

All optimization problems are defined as to minimize the induced drag. Another important concept for these problems is the elliptic lift distribution. From lifting line theory it is known that for planar wings the least induced drag is produced when there is an elliptic lift distribution along the span [43, 46]. Hence the optimal solution should have an elliptic lift distribution which helps validate our optimized results. From Fig. 5b we see that the reference wing's lift 
distribution has large differences to the elliptic distribution which indicate non-optimality.

\section{A. Choice of methods}

\section{Boundary conditions}

For the same mesh size the Dirichlet BC is approximately twice as computationally efficient as the Neumann BC [46]. This is apparent in the formulation of the influence coefficients, $\mathbf{A}_{N}, \mathbf{B}_{N}$ and $\mathbf{A}_{D}, \mathbf{B}_{D}$, where the Neumann BC requires the calculation of a vector (velocity in each direction) and the Dirichlet BC only requires a scalar (potential). As these are dense systems of equations, assembling the influence coefficient matrices is the most expensive part of the panel method accounting for roughly $80 \%$ of the computational effort. When adjoint sensitivities are calculated (through methods discussed in section IV.D $90 \%$ of the computational effort is spent on calculating sensitivities. Solutions are dependent on each point in the domain meaning that every panel in the mesh is dependent on the nodal coordinates of every other panel. Assuming quadrilateral panels, each entry of the dense matrices $\mathbf{A}$ and $\mathbf{B}$, will depend on 8 nodal points, 4 per panel (trailing edge panels have more dependencies due to the Kutta condition). This is why the sensitivity analysis dominates the computational time. Gradients are also calculated in half the time when using a Dirichlet BC compared to the Neumann $\mathrm{BC}$ due to the nature of their formulation. This leads to a substantial reduction in the computational effort required for the Dirichlet problem compared to the Neumann. Note that the influence between any two panels and their corresponding dependence on nodal points is independent of any other influence between any two panels. This means that assembly of the influence coefficients and their derivatives is easily implemented in parallel.

We also demonstrate the effect that the choice of boundary condition has on optimized designs. Consider a fixed span wing with constant NACA0012 airfoils throughout the span. The objective is to minimize the induced drag (calculated via Trefftz plane integration) subject to a lift constraint where each airfoil section has a twist variable. The problem is defined in (28) and results are shown in Fig. 6.

$$
\begin{array}{ll}
\min _{\boldsymbol{\alpha} \in \mathbb{R}^{N_{S}}} & : D_{T P}(\boldsymbol{\alpha}) \\
\text { subject to } & : L_{\mathrm{ref}}-L_{T P}(\boldsymbol{\alpha}) \leq 0 \\
& \underline{\alpha} \leq \alpha_{i} \leq \bar{\alpha} \quad \text { for } i=1, \ldots, N_{S}
\end{array}
$$

As shown in Fig. 6. Neumann and Dirichlet boundary conditions produce the same designs with a reduction in induced drag of $1.4 \%$. The design responses are a close match to the elliptic lift distribution shown in Fig. 6b. The results of this study show that both formulations create similar optimized designs, irrespective of the choice of boundary condition. Due to the computational benefits a Dirichlet BC is used for all subsequent studies. However, it is important to note that Dirichlet BC is formulated from an internal and external domain and thus requires the geometry to have a finite thickness. This means that only the Neumann BC is applicable in cases where the geometry is thin or represented 


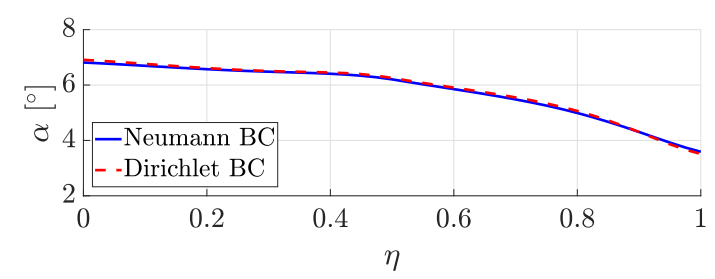

(a)

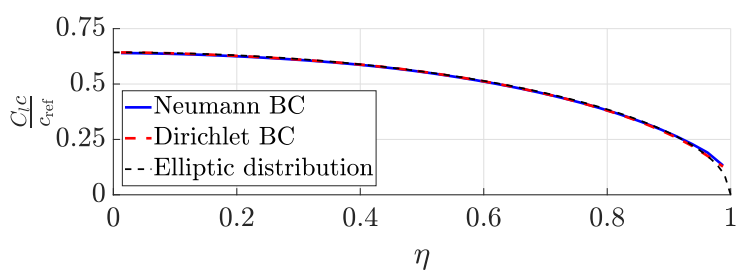

(b)

Fig. 6 Twist Optimization results: (a) twist and (b) lift distributions along the half-span of optimized designs.

by a sheet, e.g. sails, kites, etc.

\section{Force calculations}

Section [I] introduced two ways of calculating forces on the wing $-C_{P}$ and Trefftz plane integration. Both methods are appropriate for calculating the induced drag of standard or well defined wing geometries, however, results generated from $C_{P}$-integration have shown to be sensitive to the number of panels and the complexity of the wing geometry [49, 59]. The discrepancy in $C_{P}$-integration is due to cancellation errors which are of a similar magnitude to the induced drag [43]. This makes $C_{P}$-integration unreliable but it is still commonly adopted with an appropriate mesh to produce accurate results. We have found that larger differences in these methods can occur when applied to optimization problems. As an example consider the optimization problem to minimize induced drag at a fixed angle of attack of $6^{\circ}$, subject to a lift constraint where each section is defined by NACA0012 airfoils and has a chord length design variable. Optimized designs are shown in Fig. 7 where forces are calculated using $C_{P}$ and Trefftz plane integration.

$$
\begin{array}{ll}
\min _{\boldsymbol{c} \in \mathbb{R}^{N_{s}}} & : D(\boldsymbol{c}) \\
\text { subject to } & : L_{\mathrm{ref}}-L(\boldsymbol{c}) \leq 0 \\
& \underline{c} \leq c_{i} \leq \bar{c} \text { for } i=1, \ldots, N_{S}
\end{array}
$$

\begin{tabular}{ccc}
\hline Design & $D_{C_{P}} / D_{\text {ref }}$ & $D_{T P} / D_{\text {ref }}$ \\
\hline Reference & 1.004 & 1.000 \\
$\min D_{C_{P}}$ & 0.980 & 1.005 \\
$\min D_{T P}$ & 0.998 & 0.986 \\
\hline
\end{tabular}

Table 1 Drag for wings shown in Fig. 7 calculated via $C_{P}$ and Trefftz plane integration, and normalized by the drag of the reference case (calculate via Trefftz plane integration).

When using $C_{P}$-integration the design is pushed towards an unconventional geometry with a large chord at the tips. Conversely, when using Trefftz plane integration the design converges to a near elliptic wing which is known to be 

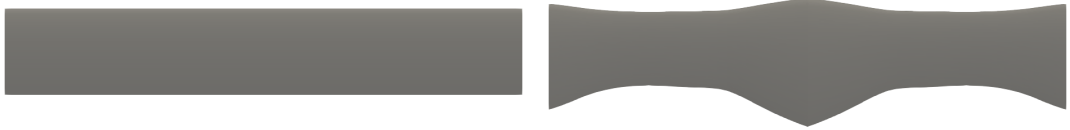

(a) Initial design/reference

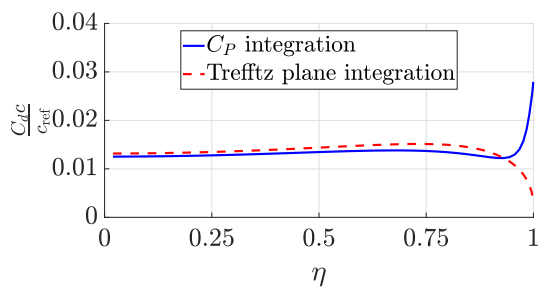

(d) Initial design/reference (b) $\min D_{C_{P}}$

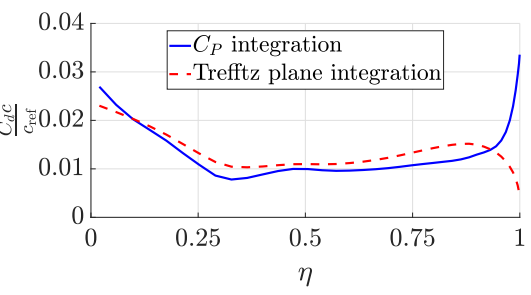

(e) $\min D_{C_{P}}$

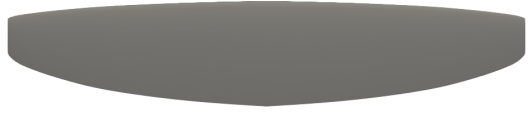

(c) $\min D_{T P}$

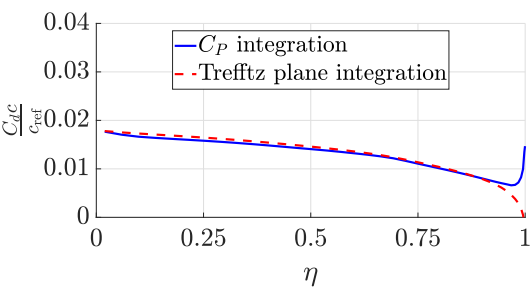

(f) $\min D_{T P}$

Fig. 7 Wing planforms and normalized sectional drag area distributions for initial and optimized designs with drag calculated from $C_{P}$ and Trefftz plane integration.

the optimal design for this problem. Results from $C_{p}$-integration tend to have numerical errors at the tip where the loads increase drastically, whereas the loads from Trefftz plane integration tend to zero towards the tip (refer to Figs. 7d 7ff. These unrealistic results can have large sensitivities that drive the optimizer to create unconventional designs that exploit the numerical error in order to decrease the objective function (refer to Table 1). Lift calculations are not as susceptible to these numerical errors as the magnitude of the error is generally much smaller than the lift. However, a dependence on the inviscid pressure distribution can still produce undesirable results for optimization problems. As the flow is inviscid, phenomena such as separation can not be predicted by the model, which can lead to non-physical $C_{P}$ distributions and an over prediction of the surface pressure. The above study suggests that $C_{P}$-integration is not suitable for optimization where large variations in geometry is permitted. In using a Trefftz plane method there is less numerical error and the optimization problem is more robust.

\section{Wake model}

Let us consider the optimization problem where the angle of attack is fixed at $6^{\circ}$ and the only variables are the chord length at each section. We seek to maximize the Oswald span efficiency factor, $e$ (minimize negative $e$ ) subject to a minimum bound on lift. According to lifting line theory, an optimal wing for this problem will have an efficiency factor of 1 which is the upper bound for planar wings. Planforms of optimized wings for various filter radii are shown in Fig. 8

$$
\begin{array}{ll}
\min _{\boldsymbol{c} \in \mathbb{R}^{N_{S}}} & :-e=-\frac{C_{L}(\boldsymbol{c})^{2}}{\pi C_{D}(\boldsymbol{c}) R(\boldsymbol{c})} \\
\text { subject to } & : L_{\mathrm{ref}}-L(\boldsymbol{c}) \leq 0 \\
& \underline{c} \leq c_{i} \leq \bar{c} \quad \text { for } i=1, \ldots, N_{S}
\end{array}
$$



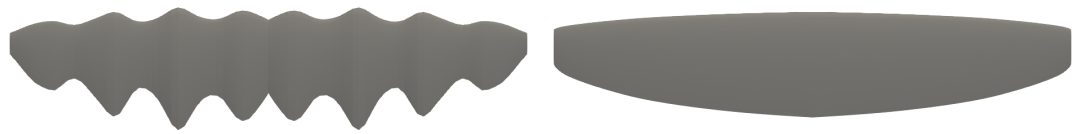
(a) $R=0$
(b) $R=\frac{b}{12}$
(c) $R=\frac{b}{6}$

Fig. 8 Planform of optimization results for the problem defined in 30 with various filter radii.

With a small filter radius more detailed features (numerical artifacts) can be created in the optimized designs. This allows the optimizer to create a fluctuating chord distribution along the span yielding wavy planform geometries (Fig. $8 \mathrm{a}$ and $8 \mathrm{~b}$. As a free-stream wake is used, the wake takes the same shape as the trailing edge of the wing projected parallel to the free-stream. The wavy structure of the wake can then create a localized upwash, reducing the drag at certain locations along the wing. The optimized designs (calculated using a free-stream wake model) in Fig. $8 \mathrm{~b}$ and $8 \mathrm{c}$ have span efficiency factors of 1.03 and 1.00 respectively. Post-analyzing the same designs using a force-free wake model (introduced in Section $[\Pi$, method presented in [41]) the span efficiencies are 0.83 and 0.98 respectively i.e. there is a large error of $24 \%$ for the wavy design (Fig. 8b) and $2 \%$ error for the elliptic design (Fig. 8c). The higher-fidelity force-free wake model captures the actual geometry of the wavy design's wake which levels out in the middle of the wake, removing the wavy-structure and subsequent upwash (see Fig. 9a and 9c). For the optimized design in Fig. 8a the force-free wake model does not converge as the wake self intersects and the calculations blow up. This study shows that realistic designs can be created using the lower-fidelity free-stream wake model provided sufficient regularization is used. The study also predicts that less regularization would be required with a force-free wake model but span-wise filtering is still needed to ensure robustness. As mentioned previously, the force-free wake model comes with a larger computational cost, therefore, only the free-stream wake model is used for optimization problems.

\begin{tabular}{ccc}
\hline Filter radius, $R$ & $e$, Free-Stream Wake & $e$, Force-Free Wake \\
\hline 0 & 1.18 & - \\
$\frac{b}{12}$ & 1.03 & 0.83 \\
$\frac{b}{6}$ & 1.00 & 0.98 \\
\hline
\end{tabular}

Table 2 Span efficiency factors for wings optimized with different filter radii. Wings are optimized with a free-stream wake model and post-analyzed designs with a force-free wake model.

\section{B. Optimization of fixed-span wings}

We will optimize the design for reducing drag under cruise conditions, where a desirable property is to have zero pitching moment, and as such we have enforced a constraint that $M_{p}=0$. From the previous discussions, we have seen that chord and twist can be used to achieve optimized designs with a similar performance. The previous designs have adopted large twist angles in order to satisfy the lift constraint. In practice large twist is generally avoided as it can lead to stall at higher angles of attack. Instead, camber is used to produce the desired lift with small values of twist. 


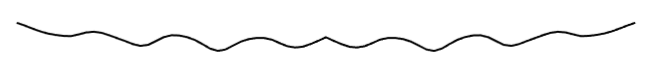

(a)

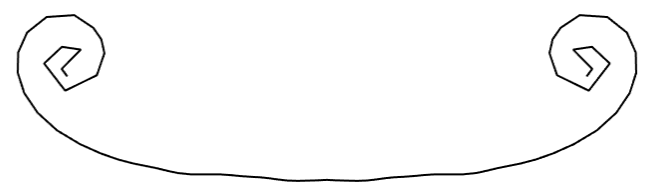

(c) (b)

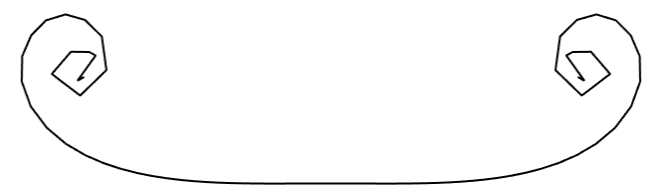

(d)

Fig. 9 Wake geometry in the Trefftz plane with (a) free-stream wake models for designs in (a) Fig. 8 b and (b) Fig. 8c; and force-free wake models for designs in (c) Fig. 8b, and (d) Fig. 8c.

The following studies include maximum bounds on twist angles but allow cambered airfoils. The design is solved with parametrization methods based on NACA 5 and B-splines airfoils. The NACA 5 parameterization is used rather than a NACA 4 because it is capable of creating a reflex camber line. This allows the airfoil sections to produce both positive and negative pitching moments without creating negative twist. The NACA 4 parameterization can only produce negative pitching moments with a negative twist and thus cannot satisfy both lift and pitching moment constraints. The design problem is defined in 31 which includes the aforementioned constraints and those discussed in Section $I V$

$$
\begin{array}{lll}
\min _{\boldsymbol{\delta} \in \mathbb{R}^{N_{\delta}}} & : D_{T P}(\boldsymbol{\delta}) & \\
\text { subject to } & : & L_{\mathrm{ref}}-L_{T P}(\boldsymbol{\delta}) \leq 0 \\
& M_{p}(\boldsymbol{\delta})=0 & \\
& \boldsymbol{\alpha}-\alpha_{\max } \leq 0 & \text { for } i=1, \ldots, N_{S} \\
& \hat{\boldsymbol{\kappa}}-\hat{\kappa}_{\max }=0 & \text { for } i=1, \ldots, N_{S} \\
& \underline{\delta} \leq \delta_{i} \leq \bar{\delta} & \text { for } i=1, \ldots, N_{\delta}
\end{array}
$$

Note that for the NACA 5 parametrization the constraint on twist, $\alpha$ is instead satisfied implicitly by the upper bound on the design variables (last constraint in (31)).

We set the upper bound on twist to be equal to the angle of attack of the reference case. Results for NACA 5 and B-spline parameterizations are shown in Fig. 10 including half-span planforms with lower and upper surface $C_{P}$, sectional lift area distributions, $C_{P}$ distributions at various $\eta$ locations, and airfoil stacks. The NACA 5 design converges to a wing of aspect ratio 9.4 and taper ratio of 0.59 . The high aspect ratio is expected, as the most effective way to reduce the induced drag is to increase $\mathbb{R}$. The B-spline parameterization has converged to a wing of aspect ratio 7.6 and taper ratio of 0.54 . The two designs have converged to similar planform geometries with an optimal elliptic lift distribution, and both produce a $1.48 \%$ reduction in total induced drag compared to the reference wing. A reflex camberline has been 
used in both cases to satisfy the pitching moment constraint and the designs produce similar $C_{P}$ distributions. The B-spline design tends to have thicker airfoils, especially towards the tip. As the flow is inviscid the airfoil thickness has minimal effect on lift or drag but we suspect that thick airfoils would be avoided if viscous effects were considered.

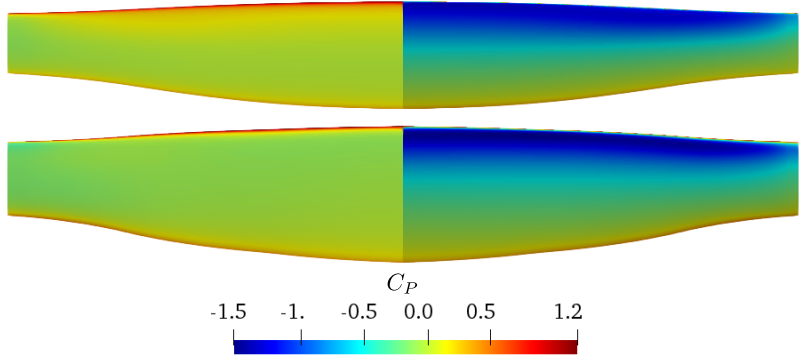

(a)

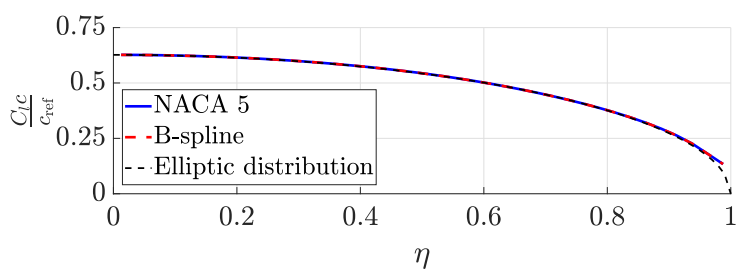

(b)

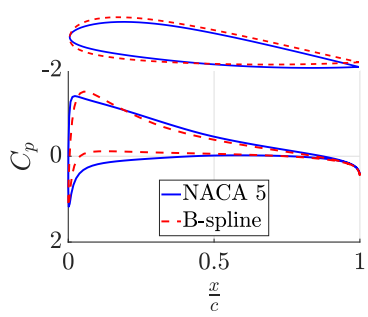

(c)

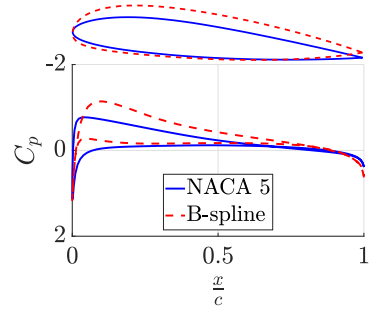

(e)

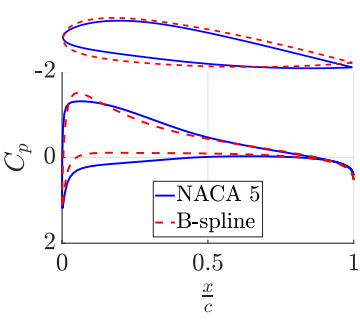

(d)

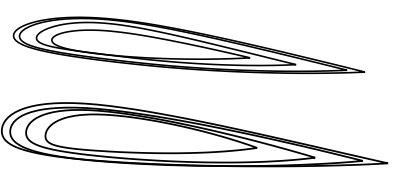

(f)

Fig. 10 (a) planforms with lower and upper surface $C_{P}$, for NACA 5 (top) and B-spline (below), (b) lift distributions, $C_{P}$ distributions and airfoils at (c) $\eta=0.05$, (d) $\eta=0.50$, (e) $\eta=0.95$, and (f) airfoil stacks with NACA-5 (top) and B-spline (below).

By taking a closer look at the individual airfoil sections we gain greater insight into the differences between the different parameterizations. Figure 11 shows the airfoil camber distributions for the NACA 5 and B-spline designs near the root, mid-section, and tip. There is a larger camber in the NACA 5 profiles and a reflex camberline is present throughout the wing. Less camber is used in B-spline profiles as there is greater freedom in the parameterization allowing the optimizer to create a reflex camberline without increasing the maximum camber. These lower cambered airfoils produce less lift. The reduction in lift (compared the the NACA 5 design) is compensated for by increasing the chord length which in-turn yields a lower aspect ratio wing. Both designs also reduce the camber from root to tip which helps achieve their elliptic lift distribution. The tip profiles of the B-spline design do not have a reflex camberline. Such airfoil sections are not possible in the NACA 5 parameterization which demonstrates the greater freedom of the B-spline parameterization.

The B-spline results presented here have shown to work well for this case. However, it took a large effort in order to get this parameterization to work efficiently. Ultimately, the B-spline parameterization has a lot more freedom to create unpredictable geometries, which can take advantage of the inviscid nature of the problem. This is because the design space is enlarged to include many possible airfoil sections that can produce the optimal span loading, thus the optimization problem is non-unique. Although it is not needed for the results presented here, we have found that these 


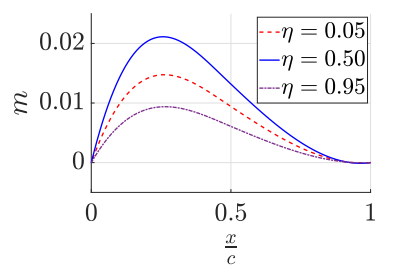

(a)

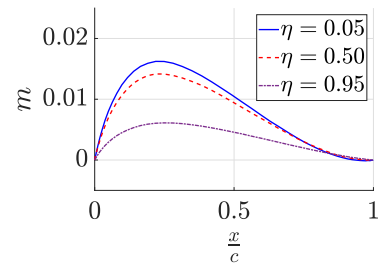

(b)

Fig. 11 Camber distributions for airfoil sections at various $\eta$ locations in the (a) NACA 5 and (b) B-spline designs

issues can be overcome by imposing additional curvature constraints or more restrictive variable bounds. However, as the motivation behind the B-spline parametrization was to enlarge the design space, it is somewhat counterproductive for it to be restricted through additional geometry constraints.

The extra freedom allowed by the B-spline parameterization method has not offered any advantage in performance over the NACA-5 parametrization. Additionally, the B-spline parameterization updates design variable bounds (as described in section III] throughout the optimization procedure in order to create a large design space whilst ensuring the spline does not self-intersect. Updating design variable bounds in this manner leads to slow convergence. Selfintersection cannot occur in the NACA parameterization methods which allows for a large design space without the need for bound updates and yields a faster convergence. Considering the increase in complexity of the problem and minimal gains in the wing's performance, the B-spline parameterization seems inappropriate for inviscid optimization problems as there is too much design freedom for the modeled physics. Accounting for viscous effects through a coupled viscous solver or finite volume methods may alleviate some of these issues experienced with the B-spline parameterization. However, this is outside the scope of this work.

\section{Optimization of variable-span wings}

Up to now we have considered wings with fixed wingspans. When it comes to induced drag minimization, the optimizer will always converge to the largest possible wingspan as this is the most effective way to reduce induced drag. Table 3 shows optimization results for the same problem defined in (31) with a NACA-5 parametrization and small perturbations to the span. The converged designs for each problem have negligible differences in their geometry, however the small changes to the wingspan has a significant effect on their induced drag. Altering the cross sections in the previous example made it possible to achieve a reduction in drag of $1.5 \%$ but as shown here if the span was $0.1 \mathrm{~m}$ shorter, the optimized design will create a drag force $1.9 \%$ higher than the reference wing. It is obvious from these results that the span has a large impact on the induced drag. This makes it difficult to improve or match the performance of the reference case with a wing of smaller span.

Wings with large wingspans are not always possible as an increased span leads to larger moments and stresses. A 


\begin{tabular}{cc}
\hline Design & $D / D_{\text {ref }}$ \\
\hline Reference & 1.000 \\
$\min D, b=6 \mathrm{~m}$ & 0.985 \\
$\min D, b=6.1 \mathrm{~m}$ & 0.953 \\
$\min D, b=5.9 \mathrm{~m}$ & 1.019 \\
\hline
\end{tabular}

Table 3 Drag of optimized designs with perturbed wingspans, each are normalized with the drag of reference case.

structural model is not considered in this work but an important physical consideration is to impose an upper bound on the wing's root bending moment defined in (7). The optimization problem for the NACA-5 parametererization is defined as

$$
\begin{aligned}
& \min _{\delta \in \mathbb{R}^{N_{\delta}}} \quad: D_{T P}(\boldsymbol{\delta}) \\
& \text { subject to } \quad: L_{\mathrm{ref}}-L(\boldsymbol{\delta}) \leq 0 \\
& M_{p}(\boldsymbol{\delta})=0 \\
& M_{R}(\boldsymbol{\delta})-M_{R, \max } \leq 0 \\
& \alpha-\alpha_{\max } \leq 0 \quad \text { for } i=1, \ldots, N_{S} \\
& \hat{\boldsymbol{\kappa}}-\hat{\kappa}_{\max }=0 \quad \text { for } i=1, \ldots, N_{S} \\
& \underline{\delta} \leq \delta_{i} \leq \bar{\delta} \quad \text { for } i=1, \ldots, N_{\delta}
\end{aligned}
$$

where $\delta$ contains an additional variable, $b$, controlling the wingspan. The maximum bound on root bending moment, $M_{R, \max }$, is defined as the root bending moment for the reference case. Results are shown in Fig. 12 for maximum bounds on span of $6 \mathrm{~m}, 7 \mathrm{~m}$, and $8 \mathrm{~m}$.

As expected, all designs have converged to the largest possible span. The design with $b_{\max }=6 \mathrm{~m}$ converges close to what we have seen previously without the bending moment constraint (Fig. 10 . Designs with larger values of $b_{\max }$ have a similar planform near the root but the chord is reduced significantly towards the tip, such that the portion of the wing for $y>3 \mathrm{~m}$ has a minimal contribution to the root bending moment. The bending moment constraint is active for each of the three designs but the constraint has a greater impact on the design for larger a span. This is also demonstrated by studying the airfoil sections and corresponding $C_{P}$ distributions where the $b_{\max }=6 \mathrm{~m}$ design is similar to the original design and large differences arise when a larger wingspan is permitted. The small airfoil sections towards the tip will in-turn lead to increased stresses and larger deformations towards the tip. This demonstrates the difficulty in producing structurally practical designs without structural coupling. When the root bending moment constraint is applied, the optimized design for a planar wing has a non-elliptic bell-shaped lifting distribution, as can be seen from Fig. 12b. These lift distributions are similar to the optimal bending moment constrained designs presented in [43], Chapter 5.10. The optimizer used a combination of increasing the outer taper and reducing twist towards the tip in order to create 


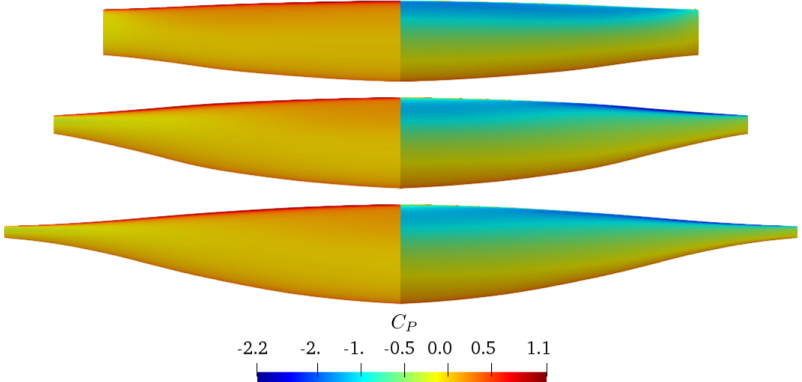

(a)

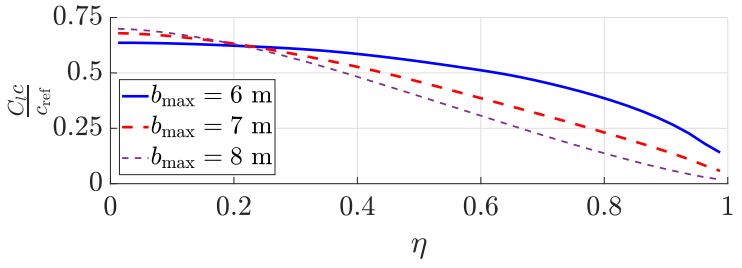

(b)

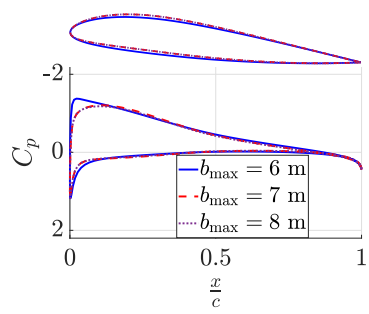

(c)

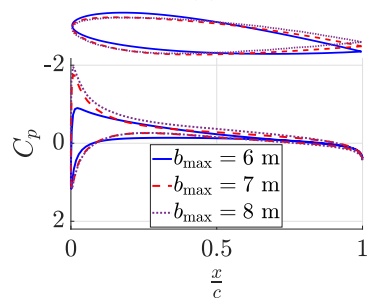

(e)

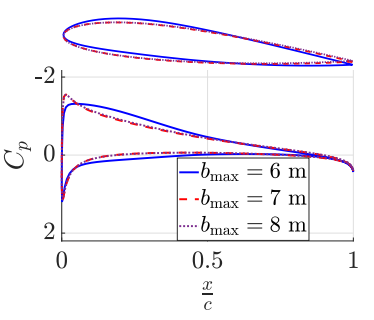

(d)

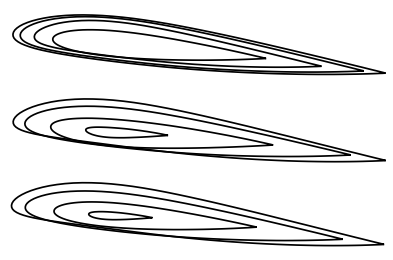

(f)

Fig. 12 (a) lower and upper surface $C_{P}$ planforms with $b_{\max }=6 \mathbf{m}$ (above), $b_{\max }=7 \mathbf{m}$ (center) and $b_{\max }=8$ m (below), (b) lift distributions, $C_{P}$ distributions and airfoils at (c) $\eta=0.05$, (d) $\eta=0.50$, (e) $\eta=0.95$, and (f) airfoil stacks with $b_{\max }=6 \mathbf{m}$ (above), $b_{\max }=7 \mathbf{m}$ (center) and $b_{\max }=8 \mathbf{m}$ (below).

these bell-shaped lift distributions.

Table 4 shows the total induced drag for designs optimized with and without the bending moment constraint. The converged designs are heavily effected by enforcing the constraint on $M_{R}$ as the drag increases drastically. However, the constrained designs are still able to provide better solutions by increasing the span. This shows the importance of structural considerations in the shape optimization of the wing.

\begin{tabular}{ccc}
\hline Design & $\begin{array}{c}D / D_{\text {ref }} \\
\text { no } M_{R} \text { constraint }\end{array}$ & $\begin{array}{c}D / D_{\text {ref }} \\
M_{R} \text { constrained }\end{array}$ \\
\hline Reference & 1.000 & 1.000 \\
$b_{\max }=6 \mathrm{~m}$ & 0.985 & 0.985 \\
$b_{\max }=7 \mathrm{~m}$ & 0.724 & 0.793 \\
$b_{\max }=8 \mathrm{~m}$ & 0.555 & 0.783 \\
\hline
\end{tabular}

Table 4 Induced drag normalized by the drag of the reference case for designs with different maximum bounds on wingspan, with and without a constraint on root bending moment, $M_{R}$.

\section{Conclusion}

Approaches to aerodynamic shape optimization of aircraft wings using panel methods have been introduced and compared by their ability to produce optimized designs. This work has found some important issues in the use of panel methods for solving optimization problems. For example, an internal Dirichlet boundary condition (BC) 
has been shown to be beneficial over an external Neumann BC as it leads to substantial cost savings for sensitivity analysis which dominates the computational effort. We have also found that calculating forces from $C_{p}$-integration is susceptible to large numerical errors that the optimizer is able to exploit in order to decrease the objective function through non-physical results. This finding suggests that $C_{p}$-integration is not suitable for induced drag calculations in optimization problems when the parameterization can allow large changes in geometry. Additionally, two methods of local parameterization are introduced, one based on the definitions of NACA 4- and 5-digit profiles, and the other using a B-spline representation, where design variables are filtered along the span to regularize the geometry. We have shown that span-wise regularization is required to prevent the optimizer from exploiting numerical weaknesses in the panel method. Both parametrization methods have proven to work well and produce similar values of the objective function, however a B-spline approach offers more design freedom than necessary for the available physics and hence requires more regularization to ensure robustness. Based on our findings we also conclude that the major challenge in using panel methods for aerodynamic optimization is to ensure that results remain physical and the optimizer is not taking advantage of the inviscid nature of the model. On this note it is expected that less regularization would be required with a model that can capture viscous effects. Finally we have demonstrated the difficulty in achieving structurally practical designs through structural considerations in the aerodynamic shape optimization problem. Future work will seek to extend the methods presented here to include a coupled structural model.

\section{Appendix}

\section{A. Verification Studies}

\section{Lifting-line theory comparison}

From lifting-line theory one can derive the following expression for the coefficient of induced drag for an elliptic wing [42, 46]

$$
C_{D}=\frac{C_{L}^{2}}{\pi R}
$$

where $\mathbb{R}$ is the wing's aspect ratio. Additionally, the coefficient of lift can be approximated as

$$
C_{L}=C_{l_{\alpha}}\left(\frac{A R}{R+2}\right) \alpha
$$

where $C_{l_{\alpha}}$ is the slope of the $2 \mathrm{D}$ lift coefficient curve from thin airfoil theory, and is approximately equal to $2 \pi$ [46]. Using these expressions we compare the results of our panel method to lifting line theory for an elliptic wing with $\mathbb{R}=5$ and comprising of symmetric airfoils.

Results from all analysis methods have a strong correlation with the lifting line theory for predicting lift at a given angle of attack. When integrating the pressure distribution with a Dirichlet boundary condition (BC) or using a Trefftz 


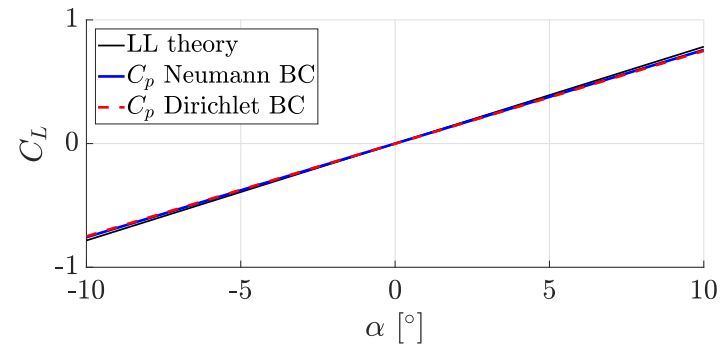

(a)

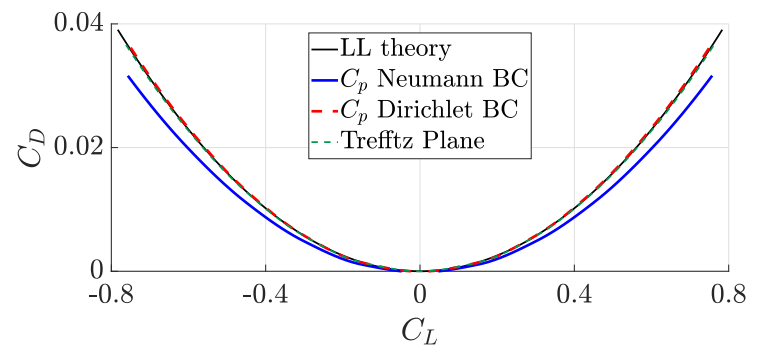

(b)

Fig. 13 Comparison between lifting-line (LL) theory and results of the panel method for an elliptic wing. Results are compared for Neumann and Dirichlet boundary conditions with $C_{P}$ integration and a Trefftz plane integration method.

plane method there is also a strong correlation in predicting the induced drag. However integrating the pressure distribution with a Neumann BC the results begin to deviate from lifting line theory for high lift. Note that the Trefftz plane integration method can be used with either BC with negligible differences between the two.

\section{VSAERO comparison}

We also compare our results with those achieved using VSAERO for three wing planforms. VSAERO calculates subsonic aerodynamic characteristics via a low-order constant source-doublet panel method [51], similar to the method implemented here. VSAERO results are achieved with a Dirichlet boundary condition and internal stagnation formulation. The first case is an unswept rectangular wing with an aspect ratio, $\mathbb{R}=5.9$. The second case is a swept wing with $\not R=5.6$, no taper, and a sweep of $20^{\circ}$. Cases 1 and 2 comprise of NACA0012 airfoils. The third case has a sweep of $45^{\circ}$, taper ratio of 0.5 , aspect ratio of 3, and NACA64A010 airfoils. Experimental and VSAERO results are taken from [60]. Figure 14 compares these results to Neumann and Dirichlet boundary conditions.

Fig. $14 \mathrm{a}$ and $14 \mathrm{~b}$ shows the local $C_{l}$ distribution with normalized semi-span, $\eta=2 y / b$ where $b$ is the wingspan. We see negligible differences in $C_{l}$ between VSAERO, Neumann and Dirichlet results, and some minor deviations compared to experimental results at the root and tip. The current work uses more panels than those presented in [60] (VSAERO) allowing our results to capture more detail around the wing tip $(\eta=1)$. In Fig. 14c the current work also matches VSAERO solutions but all numerical results diverge from the experimental as the viscous effects are excluded. There are however only small differences in the local $C_{P}$ distributions as demonstrated in Fig. 14d.

\section{SU2 comparison}

Finally we compare the panel method to results generated using SU2 [61] which is used to solve the inviscid Euler equations via a finite volume method. For this study we look at the ONERA M6 wing, which was designed in 1972 as a test case for complex flow phenomena such as transonic shocks and separated flow. Fig. 15 shows $C_{P}$ distributions for an angle of attack of $5^{\circ}$ and Mach numbers of 0.4 and 0.8 . 


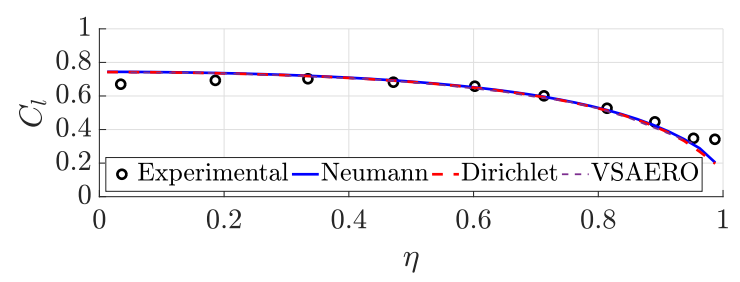

(a) Half-span lift distribution for case 1 at $\alpha=8^{\circ}$

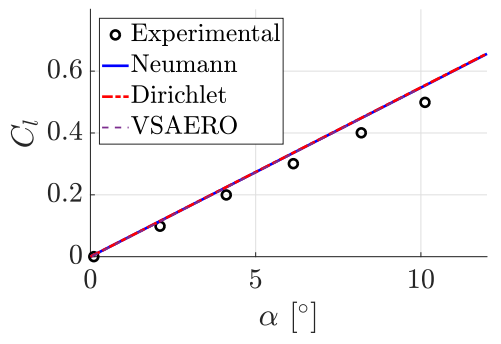

(c) Lift vs. angle of attack for case 3

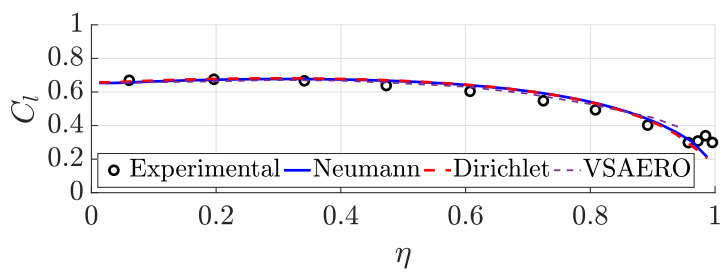

(b) Half-span lift distribution for case 2 at $\alpha=8^{\circ}$

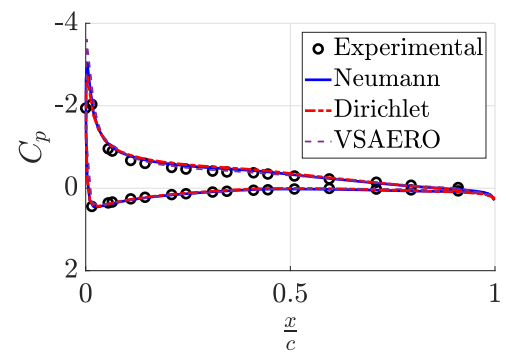

(d) $C_{P}$ distribution at $y / b=0.55$ for case 3 at $\alpha=8.23^{\circ}$

Fig. 14 Comparison between experimental, VSAERO, Neumann and Dirichlet BC results for various wing cases.

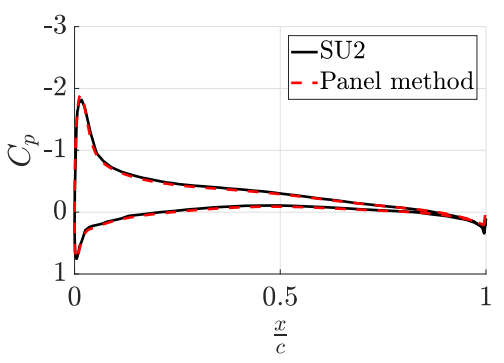

(a)

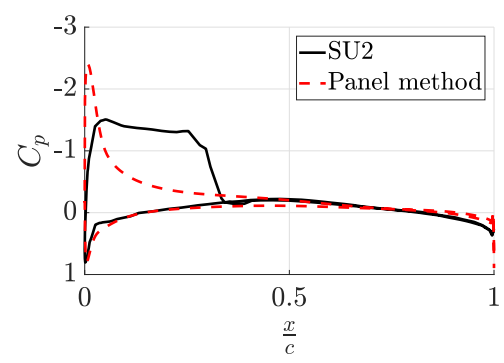

(b)

Fig. 15 Comparison between $C_{P}$ distributions at $\eta=0.5$ achieved from SU2 and panel method (current work) for the ONERA M6 wing at (a) Mach 0.4 and (b) Mach 0.8 with an angle of attack of $5^{\circ}$.

From Fig. 15 we see a good agreement between results when the flow is subsonic and a large deviation in results for transonic flows. This deviation is due to a shockwave which forms on the upper surface that the panel method is unable to predict. The inability to model shocks is one of the limitations of panel methods, however it is not an issue in this work as we are only concerned with cases where the flow is fully subsonic.

The main advantage of panel methods is their fast computational time. Table 5 compares computational times for our panel method and SU2 with single and multi-gird (3 levels) solvers using a single core. In each case the ONERA M6 wing is meshed with typical mesh sizes of 6,000 panels and roughly 500,000 finite volume elements. For the SU2 simulations the flow was converged five orders of magnitude in the density residual. The superiority of panel methods with respect to computational time can be clearly seen from these results. 


\begin{tabular}{cccc}
\hline & Panel (current) & SU2 & SU2 multi-grid (3 levels) \\
\hline Iterations & 1 & 265 & 112 \\
Time (s) & 28.6 & 1255.4 & 1037.1 \\
\hline
\end{tabular}

Table 5 Timing comparison for current panel method and SU2.

\section{Funding Sources}

The authors would like to thank the Villum Foundation for their financial support as part of the InnoTop VILLUM investigator project.

\section{References}

[1] Hess, J. L., and Smith, A. M. O., "Calculation of Potential Flow about Arbitrary Bodies," Progress in Aerospace Sciences, Vol. 8, 1967, pp. 1-138. doi:https://doi.org/10.1016/0376-0421(67)90003-6.

[2] Woodward, F. A., "Analysis and Design of Wing-Body Combinations at Subsonic and Supersonic Speeds," Journal of Aircraft, Vol. 5, No. 6, 1968, pp. 528-534. doi:https://doi.org/10.2514/3.43979.

[3] Hess, J. L., Calculation of Potential Flow about Arbitrary Three-Dimensional Lifting Bodies, Technical Report MDC J5679/01, Department of the Navy, 1972.

[4] Hicks, R. M., Murman, E. M., and Vanderplaats, G. N., An Assessment of Airfoil Design by Numerical Optimization, Technical memorandum X-3092, National Aeronautics and Space Administration, 1974.

[5] Hicks, R. M., and Vanderplaats, G. N., "Application of Numerical Optimization to the Design of Supercritical Airfoils without Drag-Creep," SAE Technical Paper 770440, 1977. doi:https://doi.org/10.4271/770440.

[6] Hicks, R. M., and Szelazel, C. A., Airfoil Design by Numerical Optimization using a Minicomputer, Technical memorandum 78502, National Aeronautics and Space Administration, 1978.

[7] Venkataraman, P., "A new procedure for airfoil definition," Proc. 13th Applied Aerodynamics Conference, American Institute of Aeronautics and Astronautics Inc., 1995. doi:https://doi.org/10.2514/6.1995-1875.

[8] Rogalsky, T., Derksen, R. W., and Kocabiyik, S., "Differential Evolution in Aerodynamic Optimization," Canadian Aeronautics and Space Journal, Vol. 46, No. 4, 2000, pp. 183-190. doi:https://doi.org/10.1.1.35.6925.

[9] Derksen, R. W., and Rogalsky, T., "Bezier-PARSEC: An Optimized Aerofoil Parameterization for Design," Advances in Engineering Software, Vol. 41, No. 7-8, 2010, pp. 923-930. doi:https://doi.org/10.1016/j.advengsoft.2010.05.002.

[10] Kyle Anderson, W., and Bonhaus, D. L., Aerodynamic Design on Unstructured Grids for Turbulent Flows, Technical memorandum 112867, National Aeronautics and Space Administration, 1997. doi:https://doi.org/10.1.1.30.2143. 
[11] Kyle Anderson, W., and Venkatakrishnan, V., "Aerodynamic Design Optimization on Unstructured Grids with a Continuous Adjoint Formulation," Computers and Fluids, Vol. 28, No. 4, 1999, pp. 443 - 480. doi:https://doi.org/10.1.1.77.7165.

[12] Nielsen, E. J., and Kyle Anderson, W., "Aerodynamic Design Optimization on Unstructured Meshes using the Navier-Stokes Equations,” AIAA Journal, Vol. 37, No. 11, 1999, pp. 1411-1419. doi:https://doi.org/10.2514/3.14337.

[13] Padula, S. L., and Li, W., "Options for Robust Airfoil Optimization under Uncertainty,” 9th AIAA/ISSMO Symposium on Multidisciplinary Analysis and Optimization, 2002. doi:https://doi.org/10.1.1.77.9271.

[14] Li, W., Huyse, L., and Padula, S., "Robust Airfoil Optimization to Achieve Drag Reduction over a Range of Mach Numbers," Structural and Multidisciplinary Optimization, Vol. 24, No. 1, 2002, pp. 38-50. doi:https://doi.org/10.1007/s00158-002-0212-4.

[15] Nemec, M., Zingg, D. W., and Pulliam, T. H., "Multipoint and Multi-Objective Aerodynamic Shape Optimization," AIAA Journal, Vol. 42, No. 6, 2004, pp. 1057-1065. doi:https://doi.org/10.2514/1.10415.

[16] Lepine, J., Trepanier, J.-Y., and Pepin, F., "Wing aerodynamic design using an optimized NURBS geometrical representation," 38th Aerospace Sciences Meeting and Exhibit, American Institute of Aeronautics and Astronautics Inc., 2000. doi:https: //doi.org/10.2514/6.2000-669.

[17] Painchaud-Ouellet, S., Tribes, C., Trépanier, J. Y., and Pelletier, D., "Airfoil Shape Optimization using a Nonuniform Rational B-splines Parameterization under Thickness Constraint,” AIAA Journal, Vol. 44, No. 10, 2006, pp. $2170-2178$. doi:https://doi.org/10.2514/1.15117.

[18] Martín, M. J., Andrés, E., Widhalm, M., Bitrián, P., and Lozano, C., "Non-Uniform Rational B-Splines-Based Aerodynamic Shape Design Optimization with the DLR TAU Code," Proceedings of the Institution of Mechanical Engineers, Part G: Journal of Aerospace Engineering, Vol. 226, No. 10, 2012, pp. 1225-1242. doi:https://doi.org/10.1177/0954410011421704.

[19] Samareh, J. A., "Aerodynamic Shape Optimization Based on Free-Form Deformation,” Proc. 10th Multidisciplinary Analysis and Optimization Conference, 2004. doi:https://doi.org/10.2514/6.2004-4630.

[20] Kenway, G. K. W., Kennedy, G. J., and Martins, J. R. R. A., “A CAD-Free Approach to High-Fidelity Aerostructural Optimization,” Proc. 13th Multidisciplinary Analysis and Optimization Conference, 2010. doi:https://doi.org/10.2514/6.2010-9231.

[21] Fornasier, L., "Wing Design Process by Inverse Potential Flow Computer Programs," AGARD Conference Proceedings No.280. the Use of Computers As a Design Tool, 1980.

[22] Hawk, J., and Bristow, D. R., "Development of MCAERO Wing Design Panel Method with Interactive Graphics Modules," NASA Contractor Report CR-3775, 1984.

[23] Chang, I. C., Van Dam, C. P., and Torres, F. J., "Wing Design Code using Three-Dimensional Euler Equations and Optimization,” Aircraft Design and Operations Meeting, American Institute of Aeronautics and Astronautics Inc., 1991. doi:https://doi.org/10.2514/6.1991-3190. 
[24] Jameson, A., Pierce, N. A., and Martinelli, L., "Optimum Aerodynamic Design using the Navier-Stokes Equations," 35th Aerospace Sciences Meeting and Exhibit, American Institute of Aeronautics and Astronautics Inc., 1997. doi:https: //doi.org/10.1.1.420.356.

[25] Reuther, J. J., Alonso, J. J., Martins, J. R. R. A., and Smith, S. C., “A Coupled Aero-Structural Optimization Method for Complete Aircraft Configurations," 37th Aerospace Sciences Meeting and Exhibit, American Institute of Aeronautics and Astronautics Inc., 1999. doi:https://doi.org/10.1.1.23.3192.

[26] Choi, S., Alonso, J. J., Kim, S., Kroo, I. M., and Wintzer, M., “Two-Level Multi-Fidelity Design Optimization Studies for Supersonic Jets," 43rd AIAA Aerospace Sciences Meeting and Exhibit, American Institute of Aeronautics and Astronautics Inc., 2005. doi:https://doi.org/10.2514/1.34362.

[27] Choi, S., Alonso, J. J., Kroo, U. M., and Wintzer, M., "Multifidelity Design Optimization of Low-Boom Supersonic Jets," Journal of Aircraft, Vol. 45, No. 1, 2008, pp. 106-118. doi:https://doi.org/10.2514/1.28948.

[28] Carmichael, R. L., and Erickson, L. L., "PANAIR - A Higher Order Panel Method for Predicting Subsonic or Supersonic Linear Potential Flows about Arbitrary Configurations," 14th Fluid and Plasma Dynamics Conference, American Institute of Aeronautics and Astronautics Inc., 1981. doi:https://doi.org/10.2514/6.1981-1255.

[29] Alonso, J. J., LeGresley, P., and Pereyra, V., "Aircraft Design Optimization,” Mathematics and Computers in Simulation, Vol. 79, No. 6, 2009, pp. 1948-1958. doi:https://doi.org/10.1016/j.matcom.2007.07.001.

[30] Rajnarayan, D., Haas, A., and Kroo, I., "A Multifidelity Gradient-Free Optimization Method and Application to Aerodynamic Design," 12th AIAA/ISSMO Multidisciplinary Analysis and Optimization Conference, American Institute of Aeronautics and Astronautics Inc., 2008, pp. 2008-6020. doi:https://doi.org/10.2514/6.2008-6020.

[31] Kennedy, G. J., and Martins, J. R. R. A., "Parallel Solution Methods for Aerostructural Analysis and Design Optimization,” 13th AIAA/ISSMO Multidisciplinary Analysis and Optimization Conference, American Institute of Aeronautics and Astronautics Inc., 2010. doi:https://doi.org/10.2514/6.2010-9308.

[32] Kennedy, G. J., and Martins, J. R. R. A., "A Parallel Aerostructural Optimization Framework for Aircraft Design Studies," Structural and Multidisciplinary Optimization, Vol. 50, No. 6, 2014, pp. 1079-1101. doi:https://doi.org/10.1007/s00158-014$1108-9$

[33] Kennedy, G. J., and Martins, J. R. R. A., "A Comparison of Metallic and Composite Aircraft Wings using Aerostructural Design Optimization," 12th AIAA Aviation Technology, Integration and Operations (ATIO) Conference and 14th AIAA/ISSMO Multidisciplinary Analysis and Optimization Conference, American Institute of Aeronautics and Astronautics Inc., 2012. doi:https://doi.org/10.2514/6.2012-5475.

[34] James, K. A., Kennedy, G. J., and Martins, J. R. R. A., “Concurrent Aerostructural Topology Optimization of a Wing Box,” Computers and Structures, Vol. 134, 2014, pp. 1-17. doi:https://doi.org/10.1016/j.compstruc.2013.12.007. 
[35] Chin, T. W., and Kennedy, G. J., "Large-Scale Compliance-Minimization and Buckling Topology Optimization of the Undeformed Common Research Model Wing," 57th AIAA/ASCE/AHS/ASC Structures, Structural Dynamics, and Materials Conference, American Institute of Aeronautics and Astronautics Inc., 2016. doi:https://doi.org/10.2514/6.2016-0939.

[36] Lambe, A. B., and Martins, J. R. R. A., "Matrix-Free Aerostructural Optimization of Aircraft Wings," Structural and Multidisciplinary Optimization, Vol. 53, No. 3, 2016, pp. 589-603. doi:https://doi.org/10.1007/s00158-015-1349-2.

[37] Kennedy, G. J., and Martins, J. R. R. A., “An Adjoint-Based Derivative Evaluation Method for Time-Dependent Aeroelastic Optimization of Flexible Aircraft,” AIAA/ASME/ASCE/AHS/ASC Structures, Structural Dynamics and Materials Conference, 2013, pp. AIAA 2013-1530. doi:https://doi.org/10.2514/6.2013-1530.

[38] Kennedy, G. J., Kenway, G. K., and Martins, J. R., “Towards Gradient-Based Design Optimization of Flexible Transport Aircraft with Flutter Constraints," 15th AIAA/ISSMO Multidisciplinary Analysis and Optimization Conference, American Institute of Aeronautics and Astronautics Inc., 2014, pp. 601-14, 601-614. doi:https://doi.org/10.2514/6.2014-2726.

[39] Goetzendorf-Grabowski, T., and Mieloszyk, J., "Common Computational Model for Coupling Panel Method with Finite Element Method," Aircraft Engineering and Aerospace Technology, Vol. 89, No. 5, 2017, pp. 654-662. doi:https://doi.org/10.1108/AEAT01-2017-0044.

[40] Mieloszyk, J., and Goetzendorf-Grabowski, T., "Introduction of Full Flight Dynamic Stability Constraints in Aircraft Multidisciplinary Optimization," Aerospace Science and Technology, Vol. 68, 2017, pp. 252-260. doi:https://doi.org/10.1016/j. ast.2017.05.024.

[41] Ramos García, N., Sørensen, J., and Shen, W., “Three-Dimensional Viscous-Inviscid Coupling Method for Wind Turbine Computations," Wind Energy, Vol. 19, No. 1, 2016, p. 67-93. doi:https://doi.org/10.1002/we.1821.

[42] Kuethe, A., and Schetzer, J., Foundations of Aerodynamics, Wiley, 1950.

[43] Drela, M., Flight Vehicle Aerodynamics, The MIT Press, 2014.

[44] Bristow, D., and Grose, G., Modification of the Douglas Neumann Program to Improve the Efficiency of Predicting Component Interference and High Lift Characteristics, NASA Contractor Report CR-3020, National Aeronautics and Space Administration, 1978.

[45] Maskew, B., "Prediction of Subsonic Aerodynamic Characteristics: A Case for Low-Order Panel Methods," Journal of Aircraft, Vol. 19, No. 2, 1982, pp. 157-163. doi:https://doi.org/10.2514/3.57369.

[46] Katz, J., and Plotkin, A., Low-Speed Aerodynamics, Cambridge Aerospace Series, Cambridge University Press, 2001.

[47] Katz, J., and Maskew, B., "Unsteady Low-Speed Aerodynamic Model for Complete Aircraft Configurations,” Journal of Aircraft, Vol. 25, No. 4, 1988, pp. 302-310. doi:https://doi.org/10.2514/3.45564.

[48] Ashley, L. D., Dudley, M. D., Iguchi, S. K., Browne, L., and Katz, J., Potential Flow Theory and Operation Guide for the Panel Code PMARC, Technical memorandum 102851, National Aeronautics and Space Administration, 1991. 
[49] Smith, S. C., A Computational and Experimental Study of Nonlinear Aspects of Induced Drag, Technical Paper 3598, National Aeronautics and Space Administration, 1996.

[50] Göthert, B., Plane and Three-Dimensional Flow at High Subsonic Speeds. (Extension of the Prandtl Rule), Technical memorandum 1105, National Advisory Commitee for Aeronautics, 1946.

[51] Maskew, B., Program VSAERO Theory Document : A Computer Program for Calculating Nonlinear Aerodynamic Characteristics of Arbitrary Configurations, NASA contractor report CR-4023, National Aeronautics and Space Administration, 1987.

[52] Erickson, L., Panel Methods - An Introduction, NASA Technical Paper 2995, National Aeronautics and Space Administration, 1990.

[53] Abbott, I. H., and von Doenhoff, A. E., Theory of Wing Sections, Dover Publications, 1959.

[54] Christensen, P. W., and Klarbring, A., "An Introduction to Structural Optimization,” Solid Mechanics and Its Applications, Vol. 153, 2008, pp. 1-220. doi:https://doi.org/10.1007/978-1-4020-8666-3.

[55] Petersson, J., and Sigmund, O., "Slope constrained Topology Optimization," International Journal for Numerical Methods in Engineering, Vol. 41, No. 8, 1998, pp. 1417-1434. doi:https://doi.org/10.1002/(sici)1097-0207(19980430)41:8<1417::aidnme344>3.0.co;2-n.

[56] Firl, M., Wüchner, R., and Bletzinger, K.-U., "Regularization of Shape Optimization Problems Using FE-based Parametrization," Struct. Multidiscip. Optim., Vol. 47, No. 4, 2013, pp. 507-521. doi:https://doi.org/10.1007/s00158-012-0843-z.

[57] Bruns, T., and Tortorelli, D., “Topology optimization of non-linear elastic structures and compliant mechanisms," Computer Methods in Applied Mechanics and Engineering, Vol. 190, No. 26-27, 2001, pp. 3443-3459. doi:https://doi.org/10.1016/s00457825(00)00278-4.

[58] Svanberg, K., “The Method of Moving Asymptotes - a New Method for Structural Optimization,” International Journal for Numerical Methods in Engineering, Vol. 24, No. 2, 1987, pp. 359-373. doi:https://doi.org/10.1002/nme.1620240207.

[59] Lee, J., "A Potential Based Panel Method for the Analysis of Marine Propellers in Steady Flow," Ph.D. thesis, Massachusetts Institute of Technology, 1987.

[60] Margason, R. J., Kjelgaard, S. O., Sellers, W. L., Morris, C. E. K., Walkley, K. B., and Shields, E. W., "Subsonic Panel Methods - A Comparison of Several Production Codes," 23rd Aerospace Sciences Meeting, American Institute of Aeronautics and Astronautics Inc., 1985. doi:https://doi.org/10.2514/6.1985-280.

[61] Economon, T. D., Palacios, F., Copeland, S. R., Lukaczyk, T. W., and Alonso, J. J., "SU2: An Open-Source Suite for Multiphysics Simulation and Design,” AIAA Journal, Vol. 54, No. 3, 2016, pp. 828-846. doi:https://doi.org/10.2514/1.J053813. 